\title{
SELF-DIFFUSION IN MAGNETICALLY ORDERED CRYSTALS
}

\section{A. Volkov}

UDC 539.219.3.001

Keywords: diffusion, ferro- and antiferromagnetic materials, vacancies.

Within the framework of the vacancy diffusion mechanism [1], the self-diffusion coefficient is defined by the expression

$$
D=\frac{a^{2}}{\tau_{0}} \cdot e^{-\frac{V}{k T}} \cdot c_{v}
$$

where $a$ is the lattice constant, $\tau_{0}$ is the time of the order of the oscillation period corresponding to the maximum frequency of the acoustic spectrum of the crystal, $V$ is the height of the potential barrier overcame by an atom, and $c_{v}$ is the equilibrium (at the given temperature) vacancy concentration in the crystal. Here the $V$ and $c_{v}$ values are determined by interatomic interaction forces; because in magnetically ordered crystals, along with the conventional Coulomb interaction, the important role is played by the exchange interaction, the special features caused by the transition to the ordered state must be observed in the diffusion coefficient for such systems. The present work is devoted to calculations of the self-diffusion coefficient for ferro- and antiferromagnetic crystals.

Let us consider at first a ferromagnetic crystal with the BCC lattice. We define the state of magnetic ordering by the spontaneous magnetization parameter $\mu$ :

$$
\mu=\frac{N_{+}-\frac{N}{2}}{N},
$$

where $N_{+}$is the number of atoms with spins oriented in the magnetization direction, and $N$ is the number of atoms in the crystal. From here for the probabilities of spin orientation along and against the magnetization direction we obtain

$$
\rho_{+}=\frac{1}{2}+\mu, \rho_{-}=\frac{1}{2}-\mu \text {. }
$$

This allows us to write down the exchange energy as follows:

$$
E_{\text {exc }}=-\frac{N z}{2} J\left(\rho_{+} \rho_{+}+\rho_{-} \rho_{-}-2 \rho_{+} \rho_{-}\right)=2 J N z \mu^{2},
$$

Ural Federal University Named after the First President of Russia B. N. El'tsin, Ekaterinburg, Russia, e-mail: vavolk@mail.ru. Translated from Izvestiya Vysshikh Uchebnykh Zavedenii, Fizika, No. 9, pp. 110-112, September, 2012. Original article submitted April 9, 2012. 
where $J$ is the exchange interaction parameter $(J>0)$ and $z$ is the coordination number. From here it follows that the energy of interatomic interaction in the crystal comprising $n$ vacancies $(n<<N)$ is

$$
E=-\frac{z v}{2} \frac{N^{2}}{N+n}-2 J z \mu^{2} \frac{N^{2}}{N+n}
$$

where $v$ is the energy of interaction of the nearest neighboring atoms taken with the minus sign. The thermodynamic probability of this state is

$$
W=\frac{(N+n) !}{N_{+} !\left(N-N_{+}\right) ! n !} .
$$

Writing down the free energy $F=E-k T W$ and minimizing it with respect to $n$, we find the equilibrium concentration of vacancies

$$
c_{V}=e^{-\frac{\frac{z v}{2}+2 J z \mu^{2}}{k T}} .
$$

As can be seen, the energy of vacancy formation below the Curie point increases at the expense of the spontaneous magnetization.

Let us calculate the value of the potential barter $V$ equal to the energy difference of the diffusing atom at points $P$ (the midpoint of the straight line section connecting the nearest central atom and the vacancy adjacent to it) and $O$ (the point of the site neighboring to the vacancy) [1]. The corresponding energies are

$$
E_{P}=-6 v-24 J \mu^{2}, E_{O}=-7 v-28 J \mu^{2} .
$$

Here we have taken into account that the distance from point $P$ to the nearest neighbors in the BCC crystal is almost equal to the distance between the first neighbors of the lattice - the difference between these distances is about $3 \%$. From Eq. (8) it follows that

$$
V=v+4 J \mu^{2}
$$

which for the diffusion coefficient yields

$$
D=D_{0} e^{-\frac{Q}{k T}},
$$

where $D_{0}=\frac{a^{2}}{\tau_{0}}$, and $Q=\frac{z+2}{2} v+4 J \mu^{2}$ is the energy of self-diffusion activation.

According to Eq. (10), for the ferromagnetic materials

$$
\ln D=\ln D_{0}-\frac{\frac{z+2}{2} v+4 J \mu^{2}}{k T},
$$




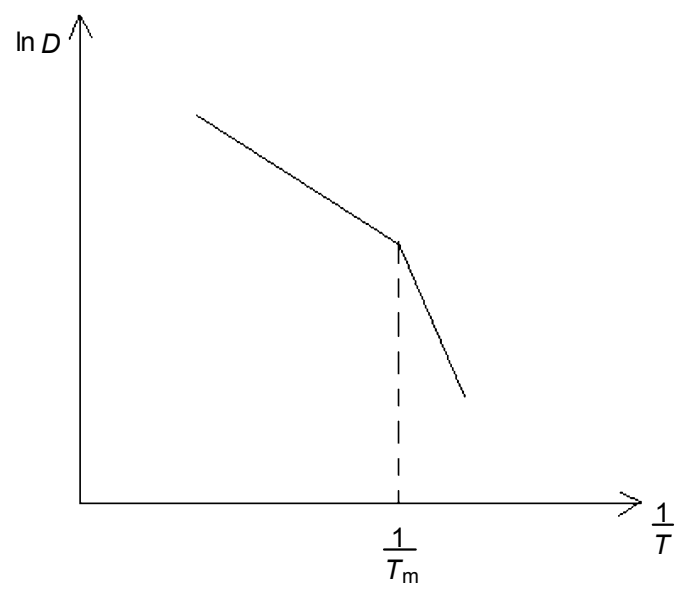

Fig. 1. Dependence of the logarithm of the self-diffusion coefficient on the reciprocal temperature for magnetically ordered crystals. Here $T_{\mathrm{m}}$ is the Curie temperature for ferromagnetic materials and the Néel temperature for antiferromagnetic materials.

that is, the Arrhenius law (the linear dependence of $\ln D$ on the reciprocal temperature) is fulfilled only for $T>T_{\mathrm{C}}$, where $T_{\mathrm{C}}$ is the Curie temperature. Below $T_{\mathrm{C}}$, the straight line illustrating the dependence of $\ln D$ on $\frac{1}{T}$ bends. The qualitative behavior of this dependence is shown in Fig. 1.

Let us now consider the self-diffusion in an antiferromagnetic BCC crystal considering that the sites in the vertices of cubes form the first magnetic sublattice and the sites in the centers of cubes form the second magnetic sublattice. Introducing the spontaneous magnetizations $\mu_{i}(i=1,2)$ of the sublattices

$$
\mu_{i}=\frac{N_{+}^{(i)}-\frac{N}{4}}{\frac{N}{2}},
$$

we can write the exchange energy as follows:

$$
E_{\text {exc }}=-2 J N z \mu_{1} \mu_{2} .
$$

For the antiferromagnetic material, $J<0$ and $\mu_{1}=-\mu_{2}=\mu$ without magnetic field, that is,

$$
E_{\text {exc }}=2 J N z \mu^{2}
$$

Calculating $c_{V}, V$, and $D$, as in the case of the ferromagnetic crystal, we arrive at Eq. (10) in which

$$
Q=\frac{z+2}{2} v-4 J \mu^{2}
$$

Thus, we have obtained analogous results both for ferro- and antiferromagnetic materials, namely, the energy of self-diffusion activation increases during transition to the magnetically ordered state. The influence of the 
magnetization on the diffusion of interstitial atoms in ferromagnetic materials was experimentally revealed in [2]. It would also be of interest to investigate the influence of magnetic ordering on the self-diffusion.

The author expresses his gratitude to Professor S. I. Masharov for useful discussions of the results obtained in this work.

\section{REFERENCES}

1. A. A. Smirnov, Molecular Kinetic Theory of Metals [in Russian], Nauka, Moscow (1966).

2. N. I. Barmin, P. V. Gel'd, V. P. Levchenko, et al., Dokl. Akad. Nauk SSSR, 215, No. 3, 567-569 (1974). 